\title{
Erratum: Defining Mononuclear Phagocyte Subset Homology across Several Distant Warm-Blooded Vertebrates through Comparative Transcriptomics
}

Frontiers Production Office*

Frontiers Production Office, Frontiers, Switzerland

Keywords: comparative biology, immunology, dendritic cells, monocytes, macrophages, genomic and bio-informatic methods

\section{An erratum on}

\section{OPEN ACCESS}

Edited and Reviewed by:

Frontiers in Immunology

Editorial Office,

Frontiers, Switzerland

*Correspondence:

Frontiers Production Office production.office@frontiersin.org

Specialty section: This article was submitted to Antigen Presenting Cell Biology,

a section of the journal

Frontiers in Immunology

Received: 01 April 2016 Accepted: 01 April 2016 Published: 28 April 2016

Citation:

Frontiers Production Office (2016)

Erratum: Defining Mononuclear Phagocyte Subset Homology across

Several Distant Warm-Blooded Vertebrates through Comparative Transcriptomics.

Front. Immunol. 7:143.

doi: 10.3389/fimmu.2016.00143
Defining Mononuclear Phagocyte Subset Homology across Several Distant Warm-Blooded Vertebrates through Comparative Transcriptomics

by Vu Manh T-P, Elhmouzi-Younes J, Urien C, Ruscanu S, Jouneau L, Bourge M, et al. Front Immunol (2015) 6:299. doi: 10.3389/fimmu.2015.00299

\section{Reason for Erratum:}

Due to a typesetting error, a misalignment in Table 1 lead to the publication of incorrect information. The publisher apologizes for this error and the correct version of Table $\mathbf{1}$ appears below.

This error does not change the scientific conclusions of the article in any way. 
TABLE 1 | Conserved gene signatures for mammalian mononuclear phagocytic cell subsets.

\begin{tabular}{ll}
$\begin{array}{l}\text { Cell subset gene } \\
\text { signatures }\end{array}$ & Genes conserved in 3/3 or 4/4 species $^{\text {a }}$ \\
\hline B cell & TRAF5; SP140; RAD17; MEF2C; MBD4; FCRL1 \\
& $\underline{\text { CD19 }}$
\end{tabular}

cDC vs. Mo/MP NAV1; MSI2; $\underline{\text { HLA-DMB; }}$ FLT3; BCL11A

Genes conserved in $2 / 3$ or $3 / 4$ species $^{b}$

cDC vs. pDC

WDR41; WDFY3; TPM4; TLR2; SPI1; SNX14; SNX10; SERPINB1; SAMHD1; RIN3; REL; RAB32; NHSL1; NCOR2; NAV1; MARCKS; LYZ; LGALS3; KLF3; JAK2; ITGA5; IL4I1; IL1B; IFNGR1; IFI30; ID2; ICAM1; HLADMB; GCA; FGL2; F11R; ETV3; DOCK7; DENND4A; CXCL16; CLEC7A; CHSY1; BATF3; ATP2B1; ARRB1; ARHGAP22; ANPEP; AIM1; AIF1; AHR; $\underline{\text { ADAM8 }}$

VPREB3; RFX5; PAX5; BACH2; AFF3; SWAP70; PLEKHA2; MS4A1; DMXL1; CR2; CD79B; CD22; BLK; ELL3; STRBP; EBF1

RFX5; PLEKHA5; HLA-DOA; BCAT2; AFF3; FAM149A; APOBEC3H; UVRAG SPINT2; PDXP; HLA-DOB; CD74; CD5; AP1S3; HLA-DRA

YWHAH; TPCN1; TDRD7; SNX21; SLC7A10; SIPA1L3; RGS12; MYO1D; MRC2; METRNL; MEA1; LRRK2; LRRC8C; LOXL3; HLA-DQB2; HAVCR2; FGF17; EHF; DOK1; DGKH; ATXN1; ASB2; ARHGAP26; ACTR3; RNF144B; PLEKHO2; MYOF; LPCAT2; KANK1; FAM114A1; DENND5A; ZNF524; VASP; SULT1A1; SPRED1; SNX8; SH3BP1; SH3BGRL; RELB; RALB; RAC1; PTPN12; PLEKHO1; PIK3CB; PAK1; NR4A1; NAB2; LFNG; JUNB; IFNGR2; IER2; HFE; FAM49B; EPSTI1; EGR1; EFHD2; DHRS3; CTBP2; COTL1; CD74; CD63; CBFB; C9ORF72; C1ORF21; BCL6; BASP1; ANXA5; SR140; PKM2; HLA-DRA; RGS4; TMSB4X; GMIP; MAST2; CXCL9; DNAJA4; KIF14; MTUS1; RABGGTA; RTN1; SYNJ1; TBX3

DCs vs. [Mo/MP and MSI2; BCL11A

$\mathrm{MoDC}]$

MoDC

TP11; NDUFV2; FCGR2B; CD200R1; ALDOA

MoDC vs. Mo/MP

TPI1; SLC2A1; SLAMF1; PRNP; PPA2; POLR1D; PLAU; PALLD; NDUFV2; NARF; MRPL4; IL1R2; FCGR2B; EGLN3; DGKA; CSNK2B; CISH; CD200R1; AVPI1; ALDOA; ADAMTSL4

\section{CEBPB; CCDC93; C5AR1}

DCs

Mo/MP vs. cDC

Mo/MP vs. MoDC

TLR4; SOD2; RBMS1; LAMP2; GLUL; FNDC3B; CYBB; CEBPB; CCPG1; CCDC93; C5AR1

WDR33; VPS13D; UBE2D2; TRA2A; STAG2; SFPQ; NSD1; NFKB1; NADK; ITPR1; CFLAR; ARFGEF1
$\mathrm{pDC}$

pDC vs. $c D C$

\section{RUNX2}

UBR2; UBE2H; TMED3; TCF4; TARBP1; SYK; STT3B; SPCS3; SNX5; SLC39A7; SIT1; SEMA4D; SEC61A1; SCYL3; SCAMP2; SAP3OBP; RUNX2; RDH11; RASGRP2; RABAC1; PPAPDC1B; PGM3; PARN; PAG1; OGT; NUCB2; MSI2; MEF2C; LMAN2; IQCB1; IFT52; HBS1L; GPAM; GORASP2; FKBP2; FAM3C; EIF2AK3; DERL1; DDOST; DAD1; CYBB; COPA; CDC42SE2; CD4; CD164; BTRC; BLNK; BCL7A; ATP2A3; ATG5
RAB34; PDCD1LG2; CHCHD7; CCL17; CARM1; AUH; VEGFA; UBA3; TUBA1A; TSKU; TMEM159; SLC48A1; SIGMAR1; RNF181; PTGR1; NOS2; IKBIP; FAM162A; BHLHE40

ZNF747; ZNF219; WIBG; VDR; SLC45A4; ROGDI; RASSF7; RAB34; RAB33A; PDE6D; PDCD1LG2; PBX2; NAGS; KCNK6; ICOSLG; HRH1; GOLGA8B; GOLGA8A; ETHE1; ERCC6; DVL2; DGUOK; CLEC1OA; CHN2; CHCHD7; CD209; CCNG2; CCL17; CARM1; C1ORF122; AUH; ANKRD37; ZEB1; VEGFA; UBA3; TUBA1A; TSKU; TMEM159; TCTEX1D2; STRA13; SPATA24; SNRNP27; SLC48A1; SIGMAR1; S1PR3; RNF181; RMND1; RAB7A; PTGR1; PIGU; PI4K2A; OST4; NSL1; NOS2; NAE1; MT1A; MORN4; LMF2; JKAMP; IKBIP; IFT46; HAUS4; GLTPD1; GATC; FAM162A; FAM13A; FAM134A; ESYT1; ERI2; EEPD1; DNLZ; DHRS11; DCTPP1; CENPW; BHLHE40; APOO; AKIP1; CD1B; CGREF1; NOSTRIN; OLFM4; GAS6; SLC27A3

TLR8; FTL; DOK3; CD68

TLR8; SNX27; RHOQ; OSTM1; KIF1B; FTL; DUSP6; DOK3; CTSD; CTSB; CD68; HERC5; IPMK; DPYD

ZNF407; VPS13C; USP31; SLC16A4; SKAP2; PRKCH; PPFIA1; PIAS2; MDN1; MAP3K5; LRRC8D; CHM; AKAP13; ACTR3; SFRS2IP; RAD51L1; NAT12; MYST3; CDC2L5; ZNF830; ZBED5; TPPP3; TMEM164; TGS1; TBC1D8B; SNRNP35; SMEK1; SLC38A10; SHISA2; RSRC2; REV1; RALGAPB; PWWP2A; PRRC2B; PBRM1; NLRC5; MOGS; MAP7D1; LUC7L3; LIMCH1; KDM4C; ISY1; IP6K1; HNRNPUL2; HNRNPU; HNRNPK; HNRNPH2; HNRNPH1; HNRNPD; HNRNPA1; FOXN3; FAM173B; FAM159B; ERVW-1; CELF2; C9; NUP210L; PDZK1; ALMS1; LAMB1; METTL3; PAIP1

LRP8; INPP4A; TSPAN13; SLC30A5; GPM6B

ZXDC; VPS13A; UEVLD; TNRC6B; TMEM63A; TAF9B; TAF1A; SUSD1; STOML1; ST6GALNAC4; SSR2; SRPRB; SPG20; SLC38A6; SLC38A1; SLC25A36; SGCB; SERPIN11; SEC24C; SAP130; RAPGEF2; RALGPS1; RAB28; RAB11FIP2; PTAR1; PIK3AP1; OSTM1; NRP1; MYB; MGAT4A; MCOLN2; MCOLN1; LRP8; KIF13B; KIAA0226; IRF7; INPP4A; IMPACT; HIVEP1; FKBP8; FANCD2; FAM122B; DMTF1; CSTF1; CREB3L2; COBLL1; CBX4; CANX; ATG4D; ANKRD28; ANKIB1; AGBL3; AFF3; TPRG1L; RNF144A; IFI27L1; FAM65B; ELMOD3; DCAF7; CARS2; ZMYND11; YPEL3; USP24; TUBGCP6; TSPAN13; TRAM1; TOE1; TMEM138; TM9SF1; TCTA; SURF4; STAMBPL1; SSR3; SPCS2; SPATA13; SNX9; SLC7A5;

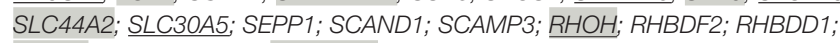
REXO2; QDPR; PYCR2; PTPRCAP; PRMT7; POLD1; PEX5; NSUN3; MTMR9; LPGAT1; INTS7; IFNAR1; HM13; GRAP; GANAB; FNDC3A; FASTK; EXOC7; ELOF1; ELMOD2; CTCF; COPE; COMMD6; CNP; CIRBP; CDS2; CD79B; CARD11; C19ORF10; C16ORF80; C10ORF88; BTD; BET1; ARHGAP12; AH11; WDR51B; SAPS3; MLF1IP; KIAA1370; CYBASC3; CEP110; CCDC111; ANUBL1; MME; PTPRS; ATF2; GPM6B; MON2; PPM1A; TM7SF3; TMCO1; UGCG; ZDHHC14; ZNF521; TMED10; PAIP1 
TABLE 1 | Continued

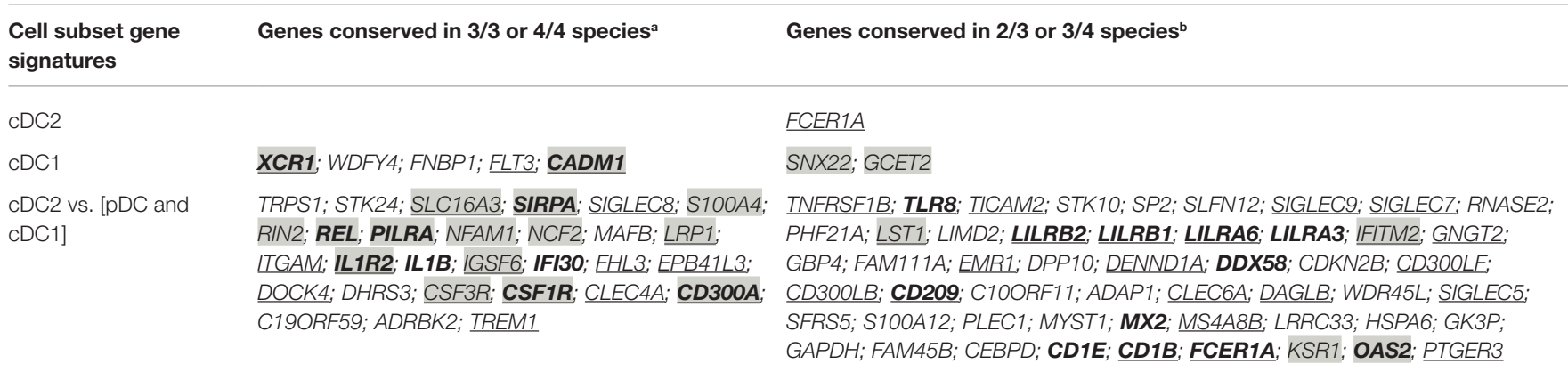

CDC1 vs. [pDC and $\quad$ XCR1; WDFY4; ST3GAL5; RAB32; PPT1; PPA1; $\mathrm{CDC}$ ]

ncMo vs. $\mathrm{CMo}^{\dagger}$

cMo vs. $\mathrm{ncMo}^{\mathrm{e}}$ ASB2; BDKRB2; BGLAP; C1ORF112; C1ORF56; NPAS2; NUB1; PCK1; PDCD4; PGR; PITPNM1; LRRC1; KIAA1598; FNBP1; FLT3; CALM1; CADM1

ACAT2; ACE; ACOT9; ADRBK2; ANKRD42; APOA2; C20ORF112; CAPZB; CBX4; CD4; CD83; $\mathrm{CDH24}$; CHD5; CSF1R; CYP2R1; DCBLD1; DDB2; DDIT4; DLGAP4; FBP1; GABBR1; GLMN; GNE; GNPNAT1; GPT; GRHPR; HEY1; HN1; IL12RB1; IL17A; IL2RG; KCNMA1; KCTD11; KNDC1; LMX1B; LUZP1; MAFF; MPZL1; MUTYH; MYOD1; NCAPH2; NCOR2; NFKBIA; PLEKHH1; PMF1; PMVK; POLR3H; RAB25; RAD52; RFC5; RHOF; RSAD1; RWDD3; SECISBP2; SERPINA1; SH2D3C; SIRT5; SLC37A1; SMS; ST3GAL1; ST3GAL5; TBC1D8; TCF7L2; TNNC1; U2AF1L4; UNG; WDR76

AACS; ABHD5; AGTPBP1; ALDH2; ALOX5AP; ANXA1; AOAH; ARL8B; ATP6V1A; ATP6V1B2; ATP6V1C1; AUH; B4GALT1; C19ORF59; C5ORF15; CCR1; CD164; CD84; CETN2; CLTA; COPB2; CSF3R; CYP27A1; DCLRE1A; DNAJC10; ECE1; EHD4; EIF2AK2; EIF2AK3; ENSA; ENTPD7; ERP29; EXOC5; F13A1; F5; FAM102B; FAM63A; FBXL5; FBXO9; FN1; GBE1; GNA12; GNPAT; GSN; GYS1; HMGB2; IL1R2; IL1RN; ITM2B; KEAP1; LACTB; LCN2; LEO1; LMAN1; LMNB1; LYZ; MBD5; MBIP; MGA; MPP1; NHLRC2; NISCH; NKRF; NPC1; NSF; NUCB2; PAM; PARP8; PDE2A; PGD; PLCB1; PNPLA8; PON2; PREPL; PRKAR1A; PRUNE; PSMA1; PSTPIP1; PUM2; PXK; PYGL; RAB27A; RAB3D; RABGAP1L; RARS; RHOT1; RMI1; RNF130; RPGR; RSC1A1; S100A8; SCRN3; SCYL1; SDCBP; SEC22C; SELL; SENP5; SERPINB1; SHB; SIGLEC1; SLC16A7; SLC25A44; SLC35B3; SLC39A9; ST8SIA4; TBC1D2; TEX2; TGM1; TM6SF1; TMEM161B; TMEM71; TPCN1; TREML2; TRIP11; TSHZ1; UBE4A; UMPS; USP10; UXS1; VAPB; VNN3; VPS37B; WDTC1; XBP1; ZMYM4
SNX22; PPAP2A; PLEKHA5; GRAMD2; DENND1B; CLEC1A; ATXN1; FAM114A1; HEPACAM2; PI4K2A; PLEKHO2; WDR91; TRIO; RALB; PKP4; PDLIM7; G3BP2; BCL6; ATPIF1; GCET2; BRWD2; FGD6; MYO9A

a Genes conserved in 4/4 species, or in 3/3 species for CDC2, cMo, and ncMo since only three species could contribute to the analysis.

${ }^{b}$ Genes conserved in 3/4 species or 2/3 species.

${ }^{\circ}$ Genes in bold were previously demonstrated to play a significant role in the development or functions of the population of interest.

"Underlined genes were annotated as located in "plasma membrane" according to Ingenuity Pathway Analysis.

'Genes highlighted in gray have been previously identified as signatures genes for the corresponding mouse and human cell populations in our earlier study (1).

fSignature genes of the relative cMo vs. ncMo and of the ncMo vs. cMo signatures were provided only for the $3 / 3$ species selection since the gene lists for the $2 / 3$ species selection encompassed hundreds of genes.

\section{REFERENCE}

1. Robbins SH, Walzer T, Dembele D, Thibault C, Defays A, Bessou G, et al. Novel insights into the relationships between dendritic cell subsets in human and mouse revealed by genome-wide expression profiling. Genome Biol (2008) 9:R17. doi:10.1186/gb-2008-9-1-r17
Copyright (c) 2016 Frontiers Production Office. This is an open-access article distributed under the terms of the Creative Commons Attribution License (CC BY). The use, distribution or reproduction in other forums is permitted, provided the original author(s) or licensor are credited and that the original publication in this journal is cited, in accordance with accepted academic practice. No use, distribution or reproduction is permitted which does not comply with these terms. 